\title{
mCRC: Capecitabin-Erhaltung nach Induktion verlängert progressionsfreies Überleben
}

\begin{abstract}
Für Patienten mit metastasiertem kolorektalem Karzinom ( $m C R C)$ ist es schwierig, nach der Induktion eine optimale Erhaltungstherapie festzulegen. Daher wurden in einer großen Phase-III-Studie Wirksamkeit und Verträglichkeit einer Erhaltungstherapie mit Capecitabin untersucht.
\end{abstract}

\begin{abstract}
$\mathrm{n}$ der randomisierten multizentrischen Studie wurden insgesamt $274 \mathrm{mCRC}$ Patienten behandelt. Nach XELOX- oder FOLFOX-Induktion über 18-24 Wochen erhielten die Patienten randomisiert entweder eine Erhaltungstherapie mit Capecitabin $(n=136)$ oder sie wurden nur beobachtet $(n=138)$. Capecitabin wurde in einer Dosierung von $1.000 \mathrm{mg} / \mathrm{m}^{2} 2$-mal täglich an Tag 1-14 q3w eingenommen.

Primärer Endpunkt der Studie war das progressionsfreie Überleben (PFS) seit Randomisierung. Sekundäre Endpunkte waren das Gesamtüberleben (OS), das PFS seit Ende der Induktionstherapie (PFS2) sowie die Verträglichkeit der Therapie.
\end{abstract}

Die Patienten im Verumarm erhielten im Median 6 Zyklen Capecitabin. Die Behandlung führte zu einem statistisch signifikant längeren $\mathrm{PFS}(6,43$ vs. 3,43 Monate; Hazard Ratio [HR] 0,54; $\mathrm{p}<0,001)$. Auch das mediane OS war in der Verumgruppe länger, jedoch ohne statistische Signifikanz zu erreichen (25,63 vs. 23,30 Monate; HR 0,85; $\mathrm{p}=0,2247)$. Hinsichtlich der Verträglichkeit waren beide Gruppen vergleichbar. Unter der Capecitabin-Erhaltung kam es jedoch häufiger zu Neutropenie, Hand-Fuß-Syndrom und Mukositis dritten Grades.

Das PFS war in allen definierten Subgruppen vergleichbar. Eine vorausgehen- de Induktionstherapie mit XELOX beeinflusste das Ergebnis der Capecitabin-Erhaltung nicht nachteilig. Patienten mit synchroner Erkrankung und mehr als einer Metastasenlokalisation scheinen jedoch stärker von der Erhaltungstherapie profitieren. Die chinesischen Patienten der Studie waren im Durchschnitt um 10 Jahre jünger als Patienten in westlichen Studien. Die guten Ergebnisse zu Effektivität und Verträglichkeit von Capecitabin sprechen aber dafür, dass das Gesamtresultat auch auf Patienten in westlichen Ländern übertragbar ist.

Fazit: Eine Capecitabin-Erhaltungstherapie kann als geeignete Option nach Induktion mit XELOX oder FOLFOX bei zuvor unbehandelten mCRC-Patienten betrachtet werden, bei insgesamt akzeptabler Verträglichkeit. Brigitte Schalhorn

Luo HY et al. Single-agent capecitabine as maintenance therapy after induction of XELOX (or FOLFOX) in first-line treatment of metastatic colorectal cancer: randomized clinical trial of efficacy and safety. Ann Oncol. 2016;27(6):107481.

\section{Radiogenomik-Nomogramm zur Vorhersage von Lymphknotenmetastasen bei CRC}

\section{Lymphknoten(Lk)-Metastasen beeinflussen Prognose und Therapie des kolorektalen Karzinoms (CRC) erheblich. Da die präoperative Computertomo- grafie hier nicht ausreichend zuverlässig ist, wurde ein Radiogenomik-Nomo- gramm entwickelt, mit dem der Lk-Befall vorausgesagt werden kann.}

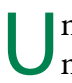
nter „Radiogenomik“ (engl. „radiomics“, eine Verschmelzung aus „radiology“ und "genomics“) versteht man die Extraktion und Analyse großer Datenmengen aus der modernen quantitativen Bildgebung (CT, PET oder MRT). Phänotypische Charakteristika (Eigenschaften wie Intensität, Gestalt und Struktur von Geweben) lassen sich so mithilfe von Algorithmen quantifizieren. Retrospektiv betrachtete man Befunde von primär 326 Patienten mit gesichertem CRC, die zwischen 2007 und 2010 in kurativer Absicht operiert worden waren. Die radiogenomischen Eigenschaften wurden extrahiert aus der portalvenösen Phase der CTs. Zunächst wurden mittels
LASSO-Regressionsmodellen die wichtigsten Eigenschaften selektiert und eine Radiogenomik-Signatur aufgebaut. Anschließend wurde das RadiogenomikNomogramm entwickelt, in das die Radiogenomik-Signatur, der Lk-Status (CT) und unabhängige klinisch-pathologische Risikofaktoren eingingen. Dieses Nomogramm wurde hinsichtlich Kalibrierung, Diskriminierungsfähigkeit und klinischem Nutzen geprüft und anschließend an weiteren 200 konsekutiven CRC-Patienten validiert.

Die Ergebnisse zeigen, dass die Radiogenomik-Signatur, bestehend aus 24 ausgewählten Eigenschaften, signifikant mit dem Lk-Status assoziiert war ( $\mathrm{p}<0,001)$.
Die Voraussagefaktoren im individualisierten Nomogramm beinhalteten $3 \mathrm{~Pa}$ rameter: Radiogenomik-Signatur, Lk-Status im CT und CEA-Spiegel. Das Modell zeigte eine gute Diskriminierung mit einem C-Index von 0,736 und eine gute Kalibrierung. Diese Eigenschaften wurden in der externen Validierung bestätigt (CIndex 0,778). Das Radiogenomik-Nomogramm erwies sich auch klinisch von Nutzen.

Fazit: Das entscheidende Argument für den klinischen Einsatz des Radiogenomik-Nomogramms ist, dass man mit seiner Hilfe im Einzelfall vorhersagen kann, ob der Patient eine zusätzliche Behandlung oder Betreuung benötigt (z. B. eine adjuvante Therapie nach der Operation). Eine Einschränkung besteht darin, dass zur Zeit der Erhebung molekulargenetische Analysen des Tumorgewebes noch nicht üblich waren.

Brigitte Schalhorn

Huang $\mathrm{Y}$ et al. Development and validation of a radiomics nomogram for preoperative prediction of lymph node metastasis in colorectal cancer. J Clin Oncol. 2016;34(18):2157-64. 